\title{
THESIS: terrestrial and habitable zone infrared spectroscopy spacecraft
}

G. Vasisht, M. R. Swain, R. L. Akeson, A. Burrows, D. Deming, et al.

G. Vasisht, M. R. Swain, R. L. Akeson, A. Burrows, D. Deming, C. J. Grillmair, T. P. Greene, "THESIS: terrestrial and habitable zone infrared spectroscopy spacecraft," Proc. SPIE 7010, Space Telescopes and Instrumentation 2008: Optical, Infrared, and Millimeter, 70103U (12 July 2008); doi: 10.1117/12.787688

SPIE Event: SPIE Astronomical Telescopes + Instrumentation, 2008, Marseille, France 


\title{
THESIS: Terrestrial and Habitable Zone Exoplanet Infrared Spectroscopy Spacecraft
}

\author{
G. Vasisht ${ }^{a}$, M. R. Swain ${ }^{a}$, R. L. Akeson ${ }^{b}$, A.Burrows ${ }^{c}$, D. Deming ${ }^{d}$ C. J. Grillmair ${ }^{b}$, T. P. \\ Greene $^{e}$ \\ ${ }^{a}$ Jet Propulsion Laboratory, California Inst. of Technology, 4800 Oak Grove Dr., Pasadena, \\ CA., USA; \\ ${ }^{b}$ Infrared Processing \& Analysis Center, California Inst. of Technology, 770 S. Wilson Ave., \\ Pasadena, CA., USA; \\ ${ }^{c}$ Dept. of Astrophysical Sciences, Princeton Univ., Princeton, N.J., USA; \\ ${ }^{d}$ Planetary Systems Laboratory, NASA Goddard Space Flight Center, Greenbelt, MD., USA; \\ ${ }^{e}$ NASA Ames Research Center, Moffet Field, CA., USA;
}

\begin{abstract}
THESIS is a concept for a medium class mission designed for spectroscopic characterization of extrasolar planets between 2-14 microns. The concept leverages off the recent first-steps made by Spitzer and Hubble in characterizing the atmospheres of alien gas giants. Under favourable circumstances, THESIS is capable of identifying biogenic molecules in habitable-zone planets, thereby determining conditions on worlds where life might exist. By systematically characterizing many worlds, from rocky planets to gas-giants, THESIS would deliver transformational science of profound interest to astronomers and the general public.
\end{abstract}

Keywords: Extrasolar Planets, Exoplanets, Atmospheres, Infrared Spectroscopy

\section{INTRODUCTION}

The last few years have witnessed astonishing progress in the area of characterization of exoplanets. While older questions regarding the frequency of occurrence of planets, and their eventual imaging etc. are still unsettled, thanks to the subclass of transiting planets - the field has evolved rapidly in characterization. This has led to a genuine mass-radius diagram for gas-giants now populated by $\sim 20$ planets, detection of direct emission, ${ }^{1-4}$ and the discovery of atmospheric features from the visible to the mid IR. Signatures of atomic species ${ }^{5}$ and aerosols in the visible, ${ }^{6}$ and molecular species in the NIR and MIR have been detected. ${ }^{7-9}$ These species govern the radiation thermodynamics of the planetary atmosphere. At the same time orbital-phase light curves using Spitzer photometry have provided testing grounds for models of radiation hydrodynamics. ${ }^{10-12}$ The extent of modulation observed in lightcurves, which reflects the differences between the day and night side brightness temperatures of the planet, has led a classification scheme for these Jovians. ${ }^{13}$ Using a combination two NASA great observatories, neither of which was designed with stable spectrophotometric capability and such science in mind, there now exists a 1.5-20 micron broadband emission spectrum for at least one planet. ${ }^{14}$ Last but not least, it has been shown that such characterization can be extended to bright planets that do not transit their parent star. ${ }^{15,16}$

Without doubt this explosive growth will continue for some time as additional planets, including superEarths that orbit close to the parent star, are discovered. Around M-dwarves, the exoplanet-to-star contrast of habitable-zone super-Earths may be favourable, allowing complete characterization of a few planets with a suitable investment of observatory exposure time from space. The number of transiting exoplanets is likely to increase dramatically over the next several years through a number of space-based (targeted, such as COROT and Kepler, and all-sky, possibly TESS) and ground-based surveys. Similarly, radial velocity surveys in the

Further author information: (Send correspondence to GV or MS)

G.V.: E-mail: gv@s383.jpl.nasa.gov, Telephone: 1-818-354-6979

M.S.: E-mail: Mark.R.Swain@jpl.nasa.gov, Telephone: 1-818-455-2396

Space Telescopes and Instrumentation 2008: Optical, Infrared, and Millimeter, edited by Jacobus M. Oschmann, Jr., Mattheus W. M. de Graauw, Howard A. MacEwen, Proc. of SPIE Vol. 7010, 70103U, (2008) $\cdot 0277-786$ X/08/\$18 $\cdot$ doi: 10.1117/12.787688

Proc. of SPIE Vol. $701070103 \mathrm{U}-1$ 
visible and now in the NIR, will find low $M \sin (i)$ planets that orbit close to the parent star - these bright planets will predominantly be non-transiting, although a small fraction, as dictated by probabilities will transit the parent star.

Transit studies are currently providing unique measurements the permit comparative (exo-)planetology. For the classes of planets where the probability of a transit is high the following can be ascertained :

- Transit photometry gives mass radius relation over a range of masses, equilibrium temperatures and orbital periods.

- Transit timing anomalies ${ }^{17}$ allows constraints on satellites and additional bodies in the planetary system.

- Rossiter-McLaughlin effect measurements ${ }^{18}$ place a bound on the stellar obliquity, which may reflect on the planet's migration history.

- Transit spectroscopy allows the study of the upper atmospheres. The slant geometry and the concomitant opacity allow observations of scatterers and absorbers at low atmospheric pressures.

- Secondary eclipse photometry allows the determination of brightness temperatures. Spectroscopy allows a better handle on atmospheric constituents, the atmospheric temperature structure etc.

- Orbital phase curves allows the mapping of horizontal temperature gradients and tests models with global circulation.

It is important to note that exoplanetary spectroscopy with a precision instrument can be extended to nontransiting planets, thereby significantly increasing the available sample. In this paper we discuss THESIS a mission dedicated to broadband exoplanet spectroscopy of both transiting and non-transiting planets. Why is such a mission needed? A powerful case can be made that only a dedicated mission can achieve the stability needed, and devote the exposure time required, to properly characterize a meaningful ensemble of planets. Although JWST will be a powerful tool for transiting planets, it is a also general astrophysics mission, with limited availability of exposure time. It is also unlikely to make an impact when the planet is not transiting because knowledge of the absolute flux of the target is lost due to primary mirror re-phasing, a variable PSF, and lack of a suitable network of flux calibrators.

\section{RESEARCH OBJECTIVES OF THESIS}

THESIS is designed to achieve what no other near-term mission can: it will characterize the nearest habitable extrasolar planet to Earth - chances are that such planet orbits a nearby M-dwarf and the orbit is in a nontransiting configuration. An overview of its science highlights includes: (i) Determining atmospheric conditions on exoplanets, including temperature structure, chemical composition, global dynamics and the relative importance of photochemistry on the highly irradiated planets. (ii) Concentrating on and determining surface conditions on a few heavy rocky planets, including temperature and chemical composition. Identifying pre-biotic molecules, such as water, methane, ammonia, hydrogen cyanide etc. and their role in atmospheric and surface chemistry. (iii) Determining system fundamental orbital parameters. Understanding the formation and evolutionary history of close-in systems. (iv) Identifying the global-scale weather and understanding the implications of meteorology on the chemistry and temperature conditions. THESIS will be unique in its ability to obtain spectra that could be compared in an absolute sense over a period of years. This capability permits measurement of orbital phaseresolved spectra (and surface mapping) of both transiting and non-transiting planets. Table 2. summarizes the kinds of measurements and science of which THESIS is capable. A more detailed consideration of hot-Jovians and habitable-zone terrestrial planets further illustrates the scientific potential.

Hot gas-giants: THESIS measurements will determine how conditions change (e.g., chemical gradients) from the dayside to the nightside of synchronously spinning planets and determine long-term changes in these conditions. For instance establishing the $\mathrm{CO} / \mathrm{CH} 4$ ratio as a function of orbital phase angle will determine the importance of photochemical and dynamical processes ${ }^{12}$ in establishing the atmospheric composition as a function of longitude. The elemental $\mathrm{C} / \mathrm{O}$ ratio, once established is an indicator of evolutionary history. Compilation 


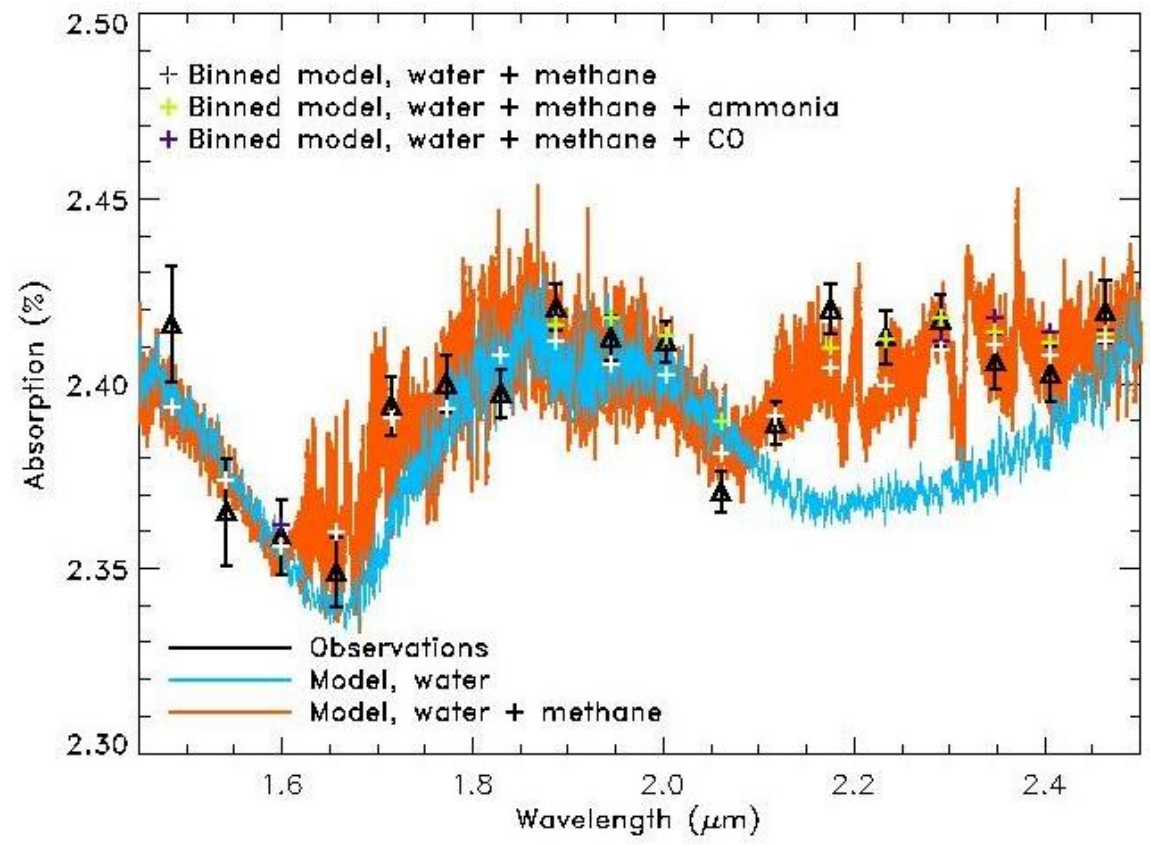

Figure 1. A HST NIR transmission spectrum of the exoplanet HD189733b taken with the G206 grism on NICMOS. Plotted alongside are theoretical transmission spectra which include collisionally induced absorption of $\mathrm{H}_{2}$, and the ro-vibrational bands of water vapor and methane. The observed data are best explained by nominal amounts of water and trace amounts of methane on this Jovian planet's terminator. ${ }^{8}$

of $\mathrm{C} / \mathrm{O}$ ratios can settle questions concerning formation and migration history. Emission lines on the dayside can reveal the presence and strength of the atmospheric temperature inversions. These measurements can build upon recent observations ${ }^{19}$ and theoretical calculations ${ }^{20,21}$ suggesting that some exoplanets form a hot stratosphere. Such measurements will significantly increase our understanding of an ensemble of hot Jovians and Neptunes. However, it is the data that THESIS could provide for large rocky planets that is truly extraordinary.

Habitable-zone terrestrial planets: A significant scientific topic for THESIS is the orbital-phase-resolved spectroscopy of a "super-Earth" orbiting an M-dwarf in the anthropomorphic liquid-water (albeit narrow) habitable zone. Characterization of atmospheric and surface conditions as a function of longitude is essential for understanding the potential for such a planet to support life. THESIS could obtain spectra as a function of orbital phase revealing properties of the atmosphere and the surface with planetary longitude. The habitable zone around an M-dwarf corresponds to $P_{\text {orb }} \sim 10-25$ days and the planets in this region are likely to be in either full or partial synchronous rotation. Tidally locked synchronous rotation does not necessarily lead to atmospheric collapse, and in such a planet a hotter dayside and a colder nightside would develop. If the difference between the two sides were truly extreme, much of the planet might not be habitable. However, if a substantial atmosphere is efficient at redistributing heat (as seems to be the case in the hot-Jovians), freeze-out could be prevented ${ }^{22}$ on the night side. For these reasons, the Astrobiology community has recently placed a strong emphasis on the common M-dwarves. ${ }^{23}$ Detailed understanding of the atmospheric chemistry obtained through THESIS measurements will address the role of photochemistry, what if any greenhouse effect is provided by the atmosphere, and the efficiency and extent of heat redistribution. As THESIS measurements can be compared on a timescale of years, the effects of weather, expected to be global on these slowly rotating worlds, ${ }^{24}$ can be determined in detail. If the planet has significant volcanism or is undergoing bombardment, THESIS measurements would likely detect these events as a departure from a long-term trend and might resolve the longitude of the event. Once again, the "super-Earth" need not transit the host star for THESIS to perform these measurements. 
Transiting
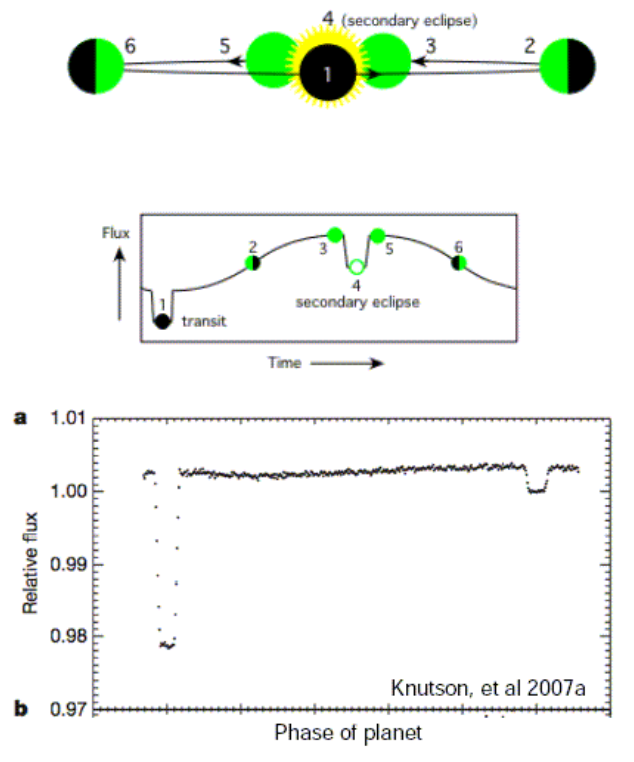

Non-transiting
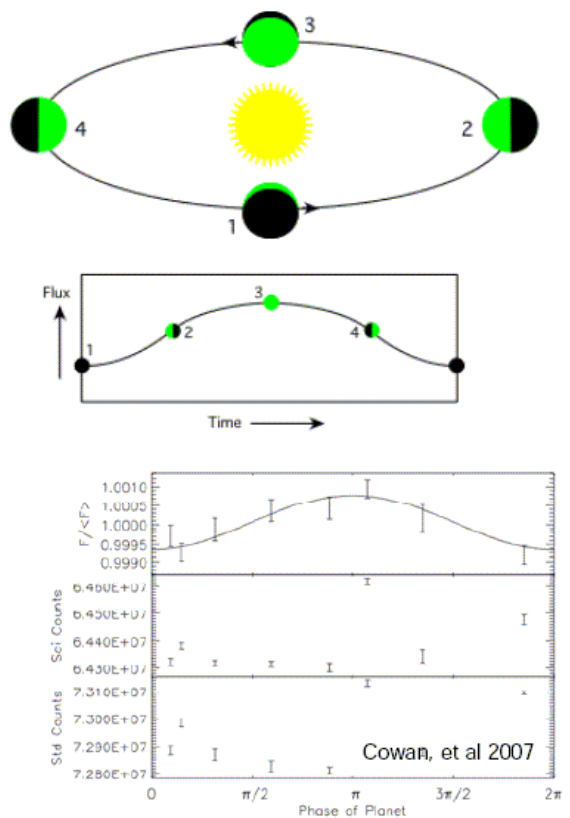

Figure 2. Schematic illustrations of observations of an exoplanet in transiting and non-transiting configurations. Left: Schematic view of a transiting system and an 8 micron lightcurve obtained with Spitzer IRAC photometry. ${ }^{10}$ Right: Schematic view of a non-transiting system and measured lightcurve. ${ }^{16}$ In both cases, THESIS would compare spectra acquired at various orbital phases. Its high stability over the course of years allows for the detection of changes in the structure of the atmosphere, or due to episodic events such as volcanism on a close in rocky planet, or bombardment.

Table 1. The THESIS Mission can be built today. This table shows the technology readiness level (TRL) for major mission critical technologies

\begin{tabular}{|l|c|l|}
\hline Mission Critical Technology & TRL & TRL Justification \\
\hline Telescope Optics and structures & 9 & Flown on numerous NASA missions \\
\hline Spectrometer Optics and structures & 9 & Flown on numerous NASA missions \\
\hline Passive cooling of telescope & 9 & Spitzer 35 K shell, expected < 40 K w/o cryogens \\
\hline Active cooling of instruments & 6 & MIRI 6K cooler TRL 6 in Dec. 2006 \\
\hline Fine Steering Mirror & 6 & Developed and tested for Space Interferometry Mission \\
\hline SWIR focal plane & 6 & Hawaii 2RG on JWST NIRspec \\
\hline MWIR focal plane & 6 & RVS 1K $\times 1$ K on JWST MIRI, TRL 6 in Jan. 2007 \\
\hline Spacecraft engineering subsystems & 9 & Kepler \& Earth orbiters; also high-end RSDO spacecraft \\
\hline
\end{tabular}

\section{THE THESIS CONCEPT}

Since THESIS observes relatively bright stars, and does not spatially separate the star-planet system, the detection of the planetary signal is limited by the shot noise from the star. In this regime the signal-to-noise is given as

$$
S N R \simeq \frac{F_{p}}{\sqrt{F_{s}}} D \sqrt{t \Delta \nu}
$$

where $F_{p}$ and $F_{s}$ denote photon fluxes from the star and planet respectively, $D$ is the telescope diameter and $t$ is the integration time. Consequentially, the SNR is proportional to telescope diameter, rather than area and the 
time required to reach a given $\mathrm{SNR}$ scales as $D^{-2}$. If the planet is at a certain contrast $\mathrm{C}$ to the star, $F_{p} \simeq C F_{s}$, the above expression becomes,

$$
S N R \simeq C \sqrt{F_{s}} D \sqrt{t \Delta \nu} .
$$

In spectrophotometry, besides the shot noise of the star, correlated noise presents a problem. This can be both astrophysical and instrumental. The former can be parameterized as fractional variability, such that the stellar flux varies by $f F_{s}$. The latter instrumental term, usually proportional in size to the incident flux, can be written as $C_{I} F_{s}$. In the presence of systematics the signal-to-noise becomes,

$$
S N R \simeq \frac{C \sqrt{F_{s}} D \sqrt{t \Delta \nu}}{\sqrt{1+\left(f^{2}+C_{I}^{2}\right)\left(F_{s} t \Delta \nu\right)}} .
$$

All this means is that 1 part in $10^{5}$ (contrast) photometry requires that astrophysical variability $(f)$ and instrumental terms $\left(C_{I}\right)$ be known to better than that. For short $t \mathrm{~s}$, lasting a few hours this should not be too hard since any IR variability on such timescales is intrinsically small and can usually be removed by observing enough points in the out of eclipse baseline using first or second order polynomials. For longer $t s$, such as a month, e.g. when observing the interrupted phase curve of a planet the problem is more difficult and requires a multi-pronged strategy. The essence of this strategy, though yet to be studied in full detail, can be itemized as follows:

- Devoting a significant fraction of the mission lifetime to observations of photometric standards that are stable to 1 part in $10^{3}$ in the visible to 1 part in $10^{4}$ in the MIR. This is done together with a low dispersion $(\mathrm{R} \sim 10)$ visible photometer and main IR spectrograph. These multicolor observations which capture the intrinsic variability of the calibrators (uncorrelated between calibrators), should allow us to derive the instrumental term in the science bands to the requisite level.

- Equipping the instrument with an internal cold (stable) source load, that can help monitor variations in the camera and detectors.

- The host stars of the planets are unlikely to be as quiet as the calibrators. Also, most of the power in the variability is concentrated on longer timescales, e.g. the rotation period of the star. The host star activity is best observed with the above mentioned visible photometer, and removed from the science (IR) data with suitable model based extrapolations.

- The above approach may not remove astrophysical activity to the desired level. In this case judicious filtering of the spectrophotometry data would help. Fortuitously, planetary variations occur would occur only on timesscales of the orbital period, $\tau_{p}$, and its harmonics. This implies that a bulk of the stellar noise in the photometry is irrelevant and only noise on timescales of $\tau_{p}, \tau_{p} / 2$ etc. matters. Such analysis would however require a well sampled orbital phase curve.

\subsection{Technology Readiness Level}

One of the great merits of THESIS is that it requires little or no new technology and can be built today. It is capable of delivering important science with a low risk approach. The beginnings and the ultimate promise of this science has now been demonstrated with both Spitzer and HST. All the hardware technology identified for such a mission is TRL 6 or higher and has either already flown or will be demonstrated on near-term mission (e.g. Kepler and JWST). Table 1 lists the mission critical technologies with current TRLs. The major mission technology areas are summarised in the next subsection.

\subsection{Technical Approach Summary}

THESIS combines $\mathrm{a} \simeq 1.5 \mathrm{~m}$ diameter parabolic primary with a hyperbolic secondary to form a f/9 telescope with an EFL of $12.6 \mathrm{~m}$ with good performance over a small by adequate 2 arcmin field of view. The primary and secondary are passively cooled to below $60 \mathrm{~K}$, reducing the thermal noise below that of the mean zodiacal background at all science wavelengths. Instruments are mounted on the back of the primary support structure. A second small parabolic mirror images the primary onto the FSM. The FSM removes spacecraft jitter to enable 


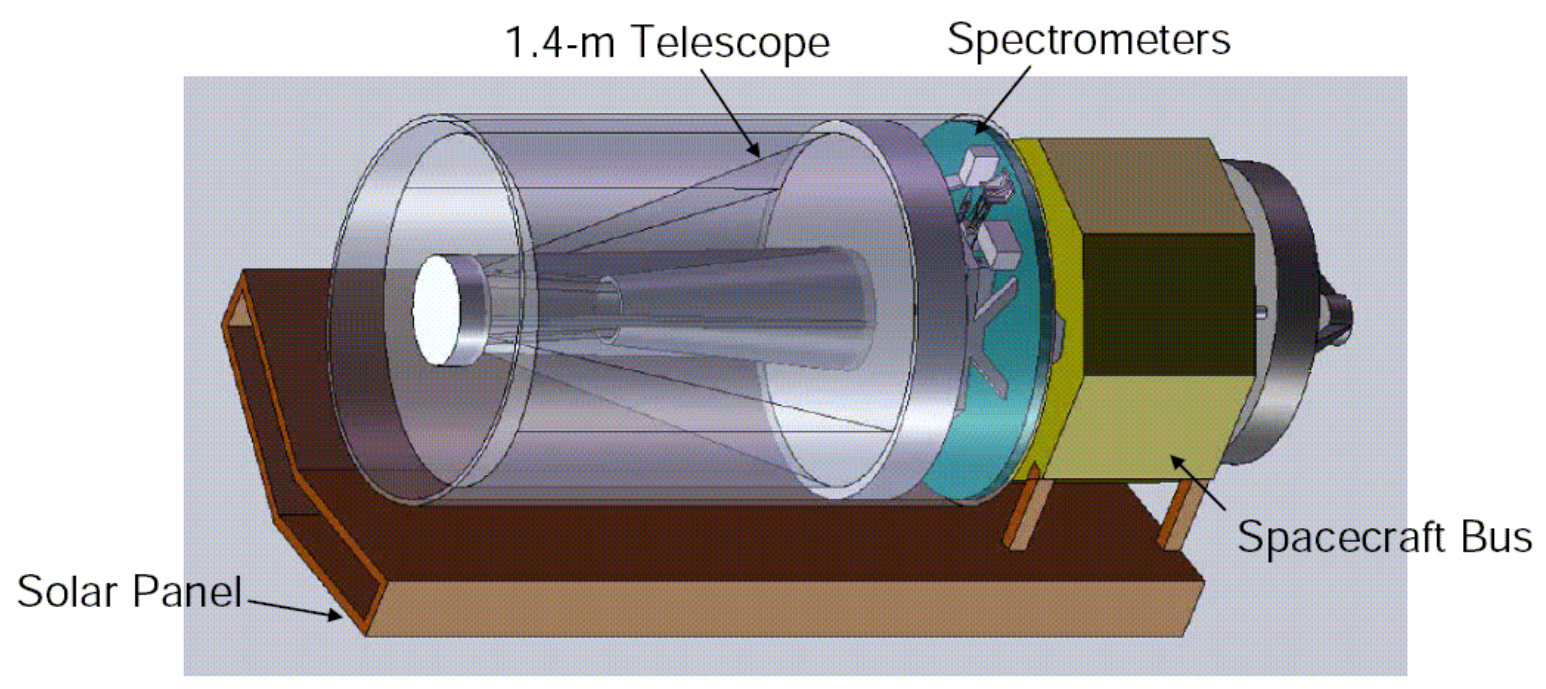

Figure 3. THESIS is an intuitively straightforward, low-risk design. A 1.5 m-diameter passively cooled telescope provides light collection for two actively cooled spectrometers, one covering 2-5 microns (SWIR) and the other 5-12 microns (MWIR). Readily available spacecraft cores possess the power, bus-level pointing and other capabilities $\mathrm{r}$ required by THESIS. A Spitzer-like solar panel/thermal shield combination makes efficient use of mass and volume resources.

on-sky pointing to better than 5 mas. The pupil is then re-imaged onto a grism dispersing element. Visible and near IR light (450-900 nm) is picked off after the FSM and imaged onto two separated CCDs, one serving as the fast guidance sensor and the other (at low spectral resolution) as a visible photometer to measure the variability of the star with crude color information. Another dichroic mirror separates the starlight into the 2-5 micron (SWIR) and 5-12 micron (MWIR) channels.

THESIS will use a combination of passive and active cryocooling to mitigate the thermal emission from the telescope. The thermal architecture will follow well understood schemes for passive cooling already demonstrated on the Spitzer spacecraft to maintain the spacecraft well below the $60 \mathrm{~K}$ level. The Spitzer telescope is expected to remain below $45 \mathrm{~K}$, with its outer shell remaining below $37 \mathrm{~K}$, after the depletion of its cryogens. Similar performance is expected for the Planck telescope, which like THESIS will use mechanical cryocoolers for its instruments. The MWIR focal plane must be cooled to $7 \mathrm{~K}$, while the SWIR camera requires cooling to $30 \mathrm{~K}$. A cryocooler of the ACDTP-type architecture can easily meet such requirements. One such cryocooler, with considerably greater capability than is required for THESIS, is currently at a high TRL level and baselined for the MIRI instrument on JWST.

As a contributor to the overall THESIS measurement stability, on-sky instrument pointing stability is currently specified at 5 mas. The requirement can be met with a multi-layer pointing control system. ${ }^{25}$ The first layer is comprised of two phases, both employing the spacecraft Attitude Control System (ACS) actuators. The normal phase uses the spacecraft star tracker as the primary control input and is characterized by multi-degree acquisition ranges and 10 arcsec pointing uncertainties.. The fine phase derives its control inputs from the CCDbased Fine Guidance Camera (FGC) within THESIS. The FGC has tens of arcsec acquisition range and supports refinement of pointing control to the 50 mas level. Several NASA missions (e.g. the Spitzer Space Telescope) have achieved this level of performance using readily available ACS components. The second layer of the pointing control system uses the FGC to provide control inputs to the FSM in the THESIS optical train. Compression of the optical beam, from a diameter of about $150 \mathrm{~cm}$ at the telescope to $3 \mathrm{~cm}$ at the FSM, results in magnification of the offsets. Thus 5 mas on the sky is about 0.25 arcsec at the FSM. Vacuum and cryo compatible, flexure mounted, voice-coil or piezo- actuated FSMs with better than 0.25 arcsec pointing resolution are comfortably within the state of the art, as is the corresponding centroiding capability required of the FGC. Accordingly, the questions to be answered regarding the second layer of THESIS pointing control are those associated with specific design and implementation choices, not technical feasibility. 
We anticipate no advancements in detector technology are needed to accomplish precision photometry required for THESIS. State of the art infrared sensor chip assemblies baselined for the JWST/NIRspec and MIRI instruments ${ }^{26}$ will be evaluated for suitability to our application. Two separate instrument focal planes will cover the entire science band. The SWIR spectrograph, covering 2-5 microns, will consider the mature $\operatorname{HgCd}_{x} \operatorname{Te}_{1-x}$ based 1K2 Hawaii-1RG array (Rockwell Teledyne ${ }^{27}$ ) to provide the high sensitivity and radiometric stability required of THESIS. Similarly, for the MWIR spectrograph we will evaluate the performance of the 1K2 Si:As Impurity Band Conduction arrays ${ }^{28}$ developed by Raytheon Vision Systems (also being used in JWST MIRI). The SWIR FPA will be cooled to a temperature similar to the optical bench (35-40 K). At these temperatures, the dark current is too low to be important. The MWIR focal planes will be operated at $7 \mathrm{~K}$ at a dark current of about $0.1 \mathrm{e} / \mathrm{s}$. For both types of detectors, a read noise of about 10 e (16 Fowler samples) at 100 kilo-pixel/s read rates is achievable. Reference pixels, which mimic dark pixels, are available on all video outputs and can be used to monitor electronic drifts. Additional immunity against effects such as interpixel capacitive coupling and intra and interpixel QE variations will be provided by optical spatial averaging.

Table 2. Measurements and Science Summary for Thesis

\begin{tabular}{|c|c|c|c|}
\hline $\begin{array}{l}\text { Measurement } \\
\text { Duration }\end{array}$ & Product & Target & Science \\
\hline \multicolumn{4}{|l|}{ Photometry } \\
\hline \multirow[t]{2}{*}{$10 \mathrm{~h}$} & Transit Lightcurve & Transit & $\begin{array}{l}\text { Planet } R, \rho \text {; weather, surface } \\
\text { features }\end{array}$ \\
\hline & Eclipse Lightcurve & Transit & Transit timing, companion detection \\
\hline $10 \mathrm{~d}$ & Orbital Lightcurve & Transit; non-Transit & $\begin{array}{l}\text { Temperature distribution } \\
\text { Atmospheric dynamics } \\
\text { Flash heated atmospheres }\end{array}$ \\
\hline \multirow[t]{2}{*}{$5 \mathrm{y}$} & Lighcurve variation & Transit & Thermal/Dynamical changes \\
\hline & Lighcurve variation & non-Transit & Thermal/Dynamical changes \\
\hline \multicolumn{4}{|l|}{ Spectroscopy } \\
\hline \multirow[t]{2}{*}{$10 \mathrm{~h}$} & Transmission spectrum & Transit & $\begin{array}{l}\text { Upper atmosphere, photochemistry } \\
\text { scattering aerosols }\end{array}$ \\
\hline & Emission spectrum & Transit & $\begin{array}{l}\text { Atmospheric chemistry 0.001-1 bar } \\
\text { P-T profiles, abundances }\end{array}$ \\
\hline \multirow[t]{3}{*}{$10 \mathrm{~d}$} & Nightside spectrum & Transit, non-Transit & $\begin{array}{l}\text { P-T profiles, molecular } \\
\text { abundance gradients }\end{array}$ \\
\hline & Terminator spectrum & Transit, non-Transit & $\begin{array}{l}\text { P-T profiles, molecular } \\
\text { abundance gradients }\end{array}$ \\
\hline & Periastron spectra & Transit, non-Transit & $\begin{array}{l}\text { flash heated atmospheres } \\
\text { layer response }\end{array}$ \\
\hline $5 \mathrm{y}$ & Spectral changes & Transit, non-Transit & $\begin{array}{l}\text { global weather changes, } \\
\text { episodic events }\end{array}$ \\
\hline
\end{tabular}

\section{ACKNOWLEDGMENTS}

This paper would not be possible without the very important contributions of several JPL technologists who have contributed to a preliminary study of the THESIS concept. The authors are grateful for contributions by A. Abramovici, R. K. Bartman, M. Jeganathan, C. G. Paine \& J. Moore. 


\section{REFERENCES}

[1] Deming, D., Seager, S., Richardson, L. J., and Harrington, J., "Infrared radiation from an extrasolar planet," Nature 434, 740-743 (Mar. 2005).

[2] Richardson, L. J., Deming, D., Horning, K., Seager, S., and Harrington, J., "A spectrum of an extrasolar planet," Nature 445, 892-895 (Feb. 2007).

[3] Grillmair, C. J., Charbonneau, D., Burrows, A., Armus, L., Stauffer, J., Meadows, V., Van Cleve, J., and Levine, D., "A Spitzer Spectrum of the Exoplanet HD 189733b," ApJ 658, L115-L118 (Apr. 2007).

[4] Swain, M. R., Bouwman, J., Akeson, R. L., Lawler, S., and Beichman, C. A., "The Mid-Infrared Spectrum of the Transiting Exoplanet HD 209458b," ApJ 674, 482-497 (Feb. 2008).

[5] Charbonneau, D., Brown, T. M., Noyes, R. W., and Gilliland, R. L., "Detection of an Extrasolar Planet Atmosphere," ApJ 568, 377-384 (Mar. 2002).

[6] Pont, F., Knutson, H., Gilliland, R. L., Moutou, C., and Charbonneau, D., "Detection of atmospheric haze on an extrasolar planet: the 0.55-1.05 $\mu \mathrm{m}$ transmission spectrum of HD 189733b with the Hubble Space Telescope," MNRAS 385, 109-118 (Mar. 2008).

[7] Tinetti, G., Vidal-Madjar, A., Liang, M.-C., Beaulieu, J.-P., Yung, Y., Carey, S., Barber, R. J., Tennyson, J., Ribas, I., Allard, N., Ballester, G. E., Sing, D. K., and Selsis, F., "Water vapour in the atmosphere of a transiting extrasolar planet," Nature 448, 169-171 (July 2007).

[8] Swain, M. R., Vasisht, G., and Tinetti, G., "The presence of methane in the atmosphere of an extrasolar planet," Nature 452, 329-331 (Mar. 2008).

[9] Charbonneau, D., Knutson, H. A., Barman, T., Allen, L. E., Mayor, M., Megeath, S. T., Queloz, D., and Udry, S., "The Broadband Infrared Emission Spectrum of the Exoplanet HD 189733b," ArXiv e-prints 802 (Feb. 2008).

[10] Knutson, H. A., Charbonneau, D., Allen, L. E., Fortney, J. J., Agol, E., Cowan, N. B., Showman, A. P., Cooper, C. S., and Megeath, S. T., "A map of the day-night contrast of the extrasolar planet HD 189733b," Nature 447, 183-186 (May 2007).

[11] Cooper, C. S. and Showman, A. P., "Dynamic Meteorology at the Photosphere of HD 209458b," ApJ 629, L45-L48 (Aug. 2005).

[12] Fortney, J. J., Cooper, C. S., Showman, A. P., Marley, M. S., and Freedman, R. S., "The Influence of Atmospheric Dynamics on the Infrared Spectra and Light Curves of Hot Jupiters," ApJ 652, 746-757 (Nov. 2006).

[13] Fortney, J. J., Lodders, K., Marley, M. S., and Freedman, R. S., "A Unified Theory for the Atmospheres of the Hot and Very Hot Jupiters: Two Classes of Irradiated Atmospheres," ApJ 678, 1419-1435 (May 2008).

[14] Swain, M. R., Vasisht, G., Tinetti, G., Bouwman, J., and Deming, D., "The Broadband Spectrum and a Chemical Gradient on the Planet HD189733b," Nature, submitted .

[15] Harrington, J., Hansen, B. M., Luszcz, S. H., Seager, S., Deming, D., Menou, K., Cho, J. Y.-K., and Richardson, L. J., "The Phase-Dependent Infrared Brightness of the Extrasolar Planet upsi Andromedae b," Science 314, 623-626 (Oct. 2006).

[16] Cowan, N. B., Agol, E., and Charbonneau, D., "Hot nights on extrasolar planets: mid-infrared phase variations of hot Jupiters," MNRAS 379, 641-646 (Aug. 2007).

[17] Agol, E., Steffen, J., Sari, R., and Clarkson, W., "On detecting terrestrial planets with timing of giant planet transits," MNRAS 359, 567-579 (May 2005).

[18] Winn, J. N., "Precise Photometry and Spectroscopy of Transits," ArXiv e-prints $\mathbf{7 1 0}$ (Oct. 2007).

[19] Knutson, H. A., Charbonneau, D., Allen, L. E., Burrows, A., and Megeath, S. T., "The 3.6-8.0 $\mu \mathrm{m}$ Broadband Emission Spectrum of HD 209458b: Evidence for an Atmospheric Temperature Inversion," ApJ 673, 526-531 (Jan. 2008).

[20] Burrows, A., Budaj, J., and Hubeny, I., "Theoretical Spectra and Light Curves of Close-in Extrasolar Giant Planets and Comparison with Data," ApJ 678, 1436-1457 (May 2008).

[21] Fortney, J. J. and Marley, M. S., "Analysis of Spitzer Spectra of Irradiated Planets: Evidence for Water Vapor?," ApJ 666, L45-L48 (Sept. 2007).

[22] Haberle, R. M., McKay, C. P., Tyler, D., and Reynolds, R. T., "Can Synchronously Rotating Planets Support An Atmosphere?," in [Circumstellar Habitable Zones], Doyle, L. R., ed., 29-+ (1996). 
[23] Tarter, J. C., Backus, P. R., Mancinelli, R. L., Aurnou, J. M., Backman, D. E., Basri, G. S., Boss, A. P., Clarke, A., Deming, D., Doyle, L. R., Feigelson, E. D., Freund, F., Grinspoon, D. H., Haberle, R. M., Hauck, II, S. A., Heath, M. J., Henry, T. J., Hollingsworth, J. L., Joshi, M. M., Kilston, S., Liu, M. C., Meikle, E., Reid, I. N., Rothschild, L. J., Scalo, J., Segura, A., Tang, C. M., Tiedje, J. M., Turnbull, M. C., Walkowicz, L. M., Weber, A. L., and Young, R. E., "A Reappraisal of The Habitability of Planets around M Dwarf Stars," Astrobiology 7, 30-65 (Mar. 2007).

[24] Rauscher, E., Menou, K., Cho, J. Y.-K., Seager, S., and Hansen, B. M. S., "Hot Jupiter Variability in Eclipse Depth," ApJ 662, L115-L118 (June 2007).

[25] Brugarolas, P. B., Kia, T., and Li, D., "Pointing control system for the eclipse mission," in [Space Telescopes and Instrumentation 1], Mather, J. C., MacEwen, H. A., and de Graauw, M. W. M., eds., Proc. SPIE 6265 (2006).

[26] Raucher, B. J. and Ressler, M., "The James Webb Space Telescope and its Infrared Detectors," Experimental Astronomy 149, 149-154 (Mar. 2005).

[27] Figer D., e. a., "Independent testing of the jwst detector prototype," in [Focal Plane Arrays for Space Telescopes], Grycewicz, T. J. and McCreight, C. R., eds., Proc. SPIE 5167, 270-301 (2004).

[28] Love, P. J., Hoffman, A. W., Lum, N. A., Ando, K. J., Rosbeck, J., Ritchie, W. D., Therrien, N. J., Holcombe, R. S., and Corrales, E., "1024 x 1024 Si:As IBC detector arrays for JWST MIRI," in [Focal Plane Arrays for Space Telescopes II. Edited by Grycewicz, Thomas J.; Marshall, Cheryl J. Proceedings of the SPIE, Volume 5902, pp. 58-66 (2005).], Grycewicz, T. J. and Marshall, C. J., eds., Presented at the Society of Photo-Optical Instrumentation Engineers (SPIE) Conference 5902, 58-66 (Aug. 2005). 